\title{
Isopropoxy Benzene Guanidine Kills Staphylococcus aureus Without Detectable Resistance
}

\author{
Xiufeng Zhang ${ }^{1,2 t}$, Wenguang Xiong ${ }^{1,2 t}$, Xianfeng Peng ${ }^{3}$, Yixing Lu ${ }^{1,2}$, Jie Hao ${ }^{1,2}$, \\ Zonghua Qin ${ }^{3}$ and Zhenling Zeng ${ }^{1,2 *}$
}

' National Risk Assessment Laboratory for Antimicrobial Resistance of Animal Original Bacteria, Guangdong Provincial Key Laboratory of Veterinary Pharmaceutics Development and Safety Evaluation, College of Veterinary Medicine, South China Agricultural University, Guangzhou, China, ${ }^{2}$ Guangdong Laboratory for Lingnan Modern Agriculture, Guangzhou, China,

${ }^{3}$ Guangzhou Insighter Biotechnology Co., Ltd., Guangzhou, China

\section{OPEN ACCESS}

Edited by:

Rodolfo García-Contreras, National Autonomous University

of Mexico, Mexico

Reviewed by:

Kui Zhu,

China Agricultural University, China Yuan Liu,

Yangzhou University, China

*Correspondence:

Zhenling Zeng

zlzeng@scau.edu.cn

${ }^{\dagger}$ These authors have contributed equally to this work

Specialty section:

This article was submitted to

Antimicrobials, Resistance

and Chemotherapy,

a section of the journal

Frontiers in Microbiology

Received: 25 November 2020

Accepted: 11 January 2021

Published: 04 February 2021

Citation:

Zhang $X$, Xiong W, Peng X, LU Y,

Hao J, Qin Z and Zeng Z (2021)

Isopropoxy Benzene Guanidine Kills

Staphylococcus aureus Without

Detectable Resistance.

Front. Microbiol. 12:633467.

doi: 10.3389/fmicb.2021.633467
Serious infections caused by multidrug-resistant Staphylococcus aureus clearly urge the development of new antimicrobial agents. Drug repositioning has emerged as an alternative approach that enables us to rapidly identify effective drugs. We first reported a guanidine compound, isopropoxy benzene guanidine, had potent antibacterial activity against $S$. aureus. Unlike conventional antibiotics, repeated use of isopropoxy benzene guanidine had a lower probability of resistance section. We found that isopropoxy benzene guanidine triggered membrane damage by disrupting the cell membrane potential and cytoplasmic membrane integrity. Furthermore, we demonstrated that isopropoxy benzene guanidine is capable of treating invasive MRSA infections in vivo studies. These findings provided strong evidence that isopropoxy benzene guanidine represents a new chemical lead for novel antibacterial agent against multidrug-resistant S. aureus infections.

Keywords: isopropoxy benzene guanidine, multidrug-resistant Staphylococcus aureus, membrane damage, resistance section, transcriptome

\section{INTRODUCTION}

Antibiotic resistance is one of the most prominent public health challenges (Årdal et al., 2019). Staphylococcus aureus is the most clinically important multidrug-resistant pathogen and a leading cause of bacteremia, endocarditis, osteomyelitis and skin, and soft tissue infections (Tacconelli et al., 2018; Turner et al., 2019). The alarming increase in the prevalence of global spread clones in S. aureus resistant to nearly all antibiotics is a major public health concern (Lakhundi and Zhang, 2018). Hence, there is a dire need to develop novel antimicrobial compounds, as well as are tolerated with low propensity for resistance development (Nambiar et al., 2014).

Nowadays, antimicrobial drug discovery is under constant challenge. The decreasing rate and huge cost of antibiotic discovery has led to alternative strategies being introduced to the clinic (Antibiotics Currently in Global Clinical Development, 2019). Repurposing drugs has emerged as an innovation stream of pharmaceutical development and gained great success in treating various infectious diseases (Austin and Gadhia, 2017; Pushpakom et al., 2019). The majority of recently approved agents against S. aureus infections have been developed from existing drug classes, including tetracycline, fluoroquinolone, and pleuromutilin (Talbot et al., 2019). In order to rescue last-resort antibiotics, researchers synthesized vancomycin derivatives by respective or combined modifications, which greatly improved 
the antibacterial activity or changed the antimicrobial mechanism (Okano et al., 2017; Guan et al., 2018).

As guanidine had strong organic bases and presented hydrophilic in nature, guanidine compounds have been discovered as new promising drugs in both synthetic and medicinal chemistry (Saczewski and Balewski, 2013; MassimbaDibama et al., 2015). Liu reported that metformin restored tetracyclines susceptibility against multidrug resistant bacteria by promoting intracellular accumulation of doxycycline (Liu Y. et al., 2020). In our previous study, we screened a guanidine compound isopropoxy benzene guanidine (IBG) as antiEnterococci agent by disrupting their cell membrane potential (Zhang et al., 2019). This compound showed low cytotoxicity against human lung epithelial cells and was well tolerated by mice red blood cells. In this study, we characterized the response of S. aureus ATCC 29213 to IBG using phenotypic assays and transcriptomics. The probability of IBG on resistance selection was estimated by serial passage assay and whole genome sequencing. The in vivo treatment efficacy of IBG was investigated in a mouse septicemia model.

\section{MATERIALS AND METHODS}

\section{Bacterial Strains, Growth Conditions}

Staphylococcus aureus strain ATCC 29213, methicillin-resistant S. aureus (MRSA) ATCC 43300, 105 clinical S. aureus isolates (including 55 MRSA strains), and 8 Gram-negative strains were used in this study. Acinetobacter baumannii and Klebsiella pneumonia were grown in Cation-Adjusted Mueller-Hinton $\mathrm{MH}$ ) broth, all other strains were grown in $\mathrm{MH}$ broth. Multilocus sequence typing (MLST) was conducted according to the reference MLST database.

\section{Antimicrobial Agents and Chemicals}

Vancomycin (VAN), gentamicin, ciprofloxacin (CIP), linezolid, and amikacin were purchased from Sangon Biotech (Shanghai, China). Isopropoxy benzene guanidine (IBG) (batch number 20150506, content 99.9\%) was synthesized by Guangzhou Insighter Biotechnology (Guangzhou, China). Dimethyl Sulphoxide (DMSO) (Dmreagent, Tianjing, China) was utilized as solvent to dissolve IBG. Anti-infective detergent benzalkonium chloride (BAC) was purchased from Aladdin Industrial Corporation (Shanghai, China). SYTOX ${ }^{\circledR}$ green nucleic acid stain agents and 3,3' -diethyloxacarbocyanine iodide $\left[\mathrm{DiOC}_{2}(3)\right]$ (Thermo Fisher Scientific, Germany) were used as molecular probes.

\section{Antibacterial Test}

The MIC and MBC of IBG were determined by broth microdilution according to CLSI guidelines (Clinical and Laboratory Standards Institute, 2018). The MBC was defined as the lowest concentration where a $99.9 \%$ colony count reduction was observed (Mohammad et al., 2015). The presence of fetal bovine serum (Tianhang Biotechnology, Zhejiang, China) on IBG activity against $S$. aureus was also tested. Experiments were performed in triplicates.

\section{Antibiotic Synergy Test}

The checkerboard method was used for determining synergy of IBG with conventional antibiotics (Falagas et al., 2019). The interaction between two compounds was defined as synergy if FICI $<0.5$, addition if $0.5 \leq$ FICI $\leq 1$, no interaction if $1<\mathrm{FICI} \leq 4$, antagonism if FICI $>4$.

\section{Killing Kinetics Assay}

The time-dependent killing for S. aureus ATCC 29213 and MRSA 43300 with IBG, VAN at $10 \times$ MIC was investigated previously (AbdelKhalek et al., 2016). The $\mathrm{OD}_{600 \mathrm{~nm}}$ was measured to determine bacterial lysis when $S$. aureus $\left(\mathrm{OD}_{600 \mathrm{~nm}} \sim 0.4\right)$ treated with $10 \times$ MIC of IBG, VAN, and BAC (positive control) for $4 \mathrm{~h}$ (Kim et al., 2018). All the experiments were replicated.

\section{Resistance Studies}

In order to select IBG-resistant mutants, $\sim 10^{10} \mathrm{CFU}$ of $S$. aureus ATCC 29213 cells were plated onto $\mathrm{MH}$ agar containing $2.5 \times, 5 \times$, and $10 \times$ MIC of IBG. After $48 \mathrm{~h}$ of incubation at $37^{\circ} \mathrm{C}$, resistant colonies were calculated and the MICs of IBG were determined. When this approach proved unsuccessful, development of resistant mutants by serial passage in liquid medium was conducted previously (Ling et al., 2015). The bacteria culture $\left(\mathrm{OD}_{600}=0.01\right)$ was treated with IBG or CIP at different concentrations. Cells were incubated at $37^{\circ} \mathrm{C}$ and passaged at $24 \mathrm{~h}$ intervals in the presence of IBG or CIP. The MIC was determined by broth microdilution. Experiments were performed with three replicates (SP1, SP2, and SP3).

The genomic DNA from the strains with elevated MIC of IBG was extracted using a Hipure bacterial DNA kit (Magen, Shanghai, China). A paired-end sequencing library $(2 \mathrm{bp} \times 250 \mathrm{bp})$ was created using a VAHTS Universal DNA Library Prep kit for Illumina ${ }^{\circledR}$ (Illumina, San Diego, CA, United States) and sequenced on an Illumina HiSeq system (Illumina Inc.). Processed reads were de novo assembled into draft genomes using CLC Genomics Workbench 10.1 (CLC Bio, Aarhus, Denmark) and annotated by using Prokka pipeline. These genomes were subjected to SNP analysis by utilizing snippy pipeline. S. aureus ATCC 29213 genome of the starting strain was used as a reference genome in SNP analysis.

\section{Membrane Potential Assay}

To examine the perturbation of the cell membrane of $S$. aureus by IBG, the membrane potential of the cells was measured by fluorescence spectrometry using fluorescent probe $\operatorname{DiOC}_{2}(3)$, as described previously (Wang et al., 2017). Bacterial cells were energized by the addition of glucose to establish a proton motive force (negative and basic inside the cell). This led to an increase in fluorescence associated with aggregation of the $\mathrm{DiOC}_{2}(3)$. Upon addition of the ionophore CCCP, the $\Delta \psi$ was dissipated and the fluorescence intensity dropped to the level before addition of glucose. All assays were performed at least twice.

\section{Membrane Permeability Assay}

To confirm the integrity of the bacterial membranes, we performed an assay based on the uptake of the fluorescent 
dye with SYTOX Green (Kim et al., 2018). The fluorescence was measured using a multifunctional microplate reader, with excitation and emission wavelengths of 485 and $525 \mathrm{~nm}$, respectively. All experiments were conducted in duplicate.

\section{Transmission Electron Microscopy}

Morphological appearance of the cell membrane of S. aureus (ATCC 29213) treated with $10 \times$ MIC IBG was observed using a JEOL 1200EX transmission electron microscopy (TEM) (Li et al., 2016).

\section{Transcriptome Analysis}

Staphylococcus aureus ATCC 29213 cells $\left(\mathrm{OD}_{600} \sim 0.4\right)$ were treated with $10 \times$ MIC IBG for $4 \mathrm{~h}$. Then bacteria was collected and preserved with RNA protect (Qiagen, United States). Total RNA of each sample was extracted using TRIzol Reagent (Invitrogen)/RNeasy Mini Kit (Qiagen). Control samples were collected from an antibiotic-free culture. RNA sequencing was conducted by High-Throughput Sequencing Facility at the GENWIZ lnc (Jiangsu, China). Raw sequence data were underwent quality control using Cutadapt (V1.9.1) and FastQC (V0.10.1). Clean data were aligned with the reference genome of S. aureus NCTC 8325 (NCBI accession number NC_007795.1) via software Bowtie2 (v2.1.0) and the gene expression level were estimated by HTSeq (v0.6.1p1). The calculation of fragment per kilobase of exon per million fragments mapped (FPKM) for all genes were performed through Cufflinks (v2.2.1) software. Differential expression genes (DEGs) were screened out by using the DESeq Bioconductor package and defined as those with a change in expression of $>$ twofold and a corresponding false discovery rate (FDR) of $<0.05$. The gene ontology terms and functional pathways were annotated via Gene Ontology (GO) and Kyoto Encyclopedia of Genes and Genome (KEGG), respectively. Cell-PLoc 2.0 was used to analyze the subcellular localization of DEGs (Chou and Shen, 2010).

\section{Mouse Sepsis Protection Model}

Animal studies were carried out at animal laboratory center of South China Agricultural University and approved by the Animal Research Committee of South China Agricultural University (2018030). All animal studies were performed with specificpathogen-free female KM mice (Southern Medical University, Guangdong, China), 6-8-weeks old, weighing $20 \pm 2$ g.

A mouse septicemia protection assay was used to assess in vivo treatment efficacy of IBG (Thangamani et al., 2016). KM female mice were treated intraperitoneally with a dose of $40 \mathrm{mg} / \mathrm{kg}$ IBG. After $24 \mathrm{~h}$, KM female mice were infected with $0.1 \mathrm{ml}$ of bacterial suspension (MRSA YXMC004P) via tail vein injection, a concentration that achieves about $90 \%$ mortality within 18 days after infection. At $0.5 \mathrm{~h}$ and $24 \mathrm{~h}$ post infection, mice (18 per group) were treated with $40 \mathrm{mg} / \mathrm{kg}$ IBG, $15 \mathrm{mg} / \mathrm{kg}$ VAN and PBS via intraperitoneal injection. Mice were monitored for 18 days after MRSA infection and the statistical analysis was performed by non-parametric log-rank test.

\section{Statistical Analyses}

Statistical analysis was performed using GraphPad Prism 5 and SPSS software. All data were presented as the mean \pm s.d.

\section{Data Availability}

The whole-genome resequencing for eight $S$. aureus strains involved in resistance studies have been deposited in GenBank under accession numbers: VUKQ00000000, VUKK00000000, VUKL00000000, VUKM00000000, VUKO00000000, VUKP00000000, VUKR00000000, and VUKS00000000. RNA-seq data have been deposited in the NCBI's Sequence Read Archive with accession number PRJNA557004.

\section{RESULTS}

\section{Antimicrobial Activity of IBG}

The structure of IBG was shown in Figure 1A. IBG exhibited potent activity against all tested $S$. aureus with the MIC range of $0.125-4 \mu \mathrm{g} / \mathrm{ml}$ (Table 1). However, IBG was inactive against Gram-negative bacteria (Supplementary Table 1). Besides, in the presence of $10 \%$ FBS, the MIC range increased eightfold. The $\mathrm{MBC}$ range for IBG against $S$. aureus strains harboring different MLST types was 4-8-fold of their MICs (Table 2). The kill kinetics of IBG was similar to VAN, which greatly reduced the number of bacteria within $4 \mathrm{~h}$ (Figures 1B,C). However, IBG and VAN did cause $S$. aureus lysis (Figure 1D). In addition, IBG exhibited addition activity $(0.5<$ FICI $<1)$ with gentamicin or amikacin when tested against S. aureus ATCC 29213, MRSA ATCC 43300 (Supplementary Figure 1).

\section{IBG Had a Low Probability of Resistance Selection in S. aureus}

We were unable to obtain IBG-resistant mutants by plating $10^{10} \mathrm{CFU}$ of $S$. aureus ATCC 29213 on agar containing $2.5 \times$, $5 \times$ or $10 \times$ MIC of IBG. Similarly, serial passage of three independent $S$. aureus 29,213 cultures for 100 days treated with IBG yielded only putative mutants with two or fourfold greater resistance to IBG, whereas serial passage in CIP for 100 days generated strains that were 512-fold more resistant (Figure 1E and Table 3). A total of 24 mutation genes were identified and most mutations genes encoded products related to catalytic activity, binding and proton transmembrane transporter activity (Supplementary Table 2). In addition, atpE, $p c r A$, and walR genes were responsible for the reduced susceptibility of IBG.

\section{IBG Disrupted Cell Membrane in Multiple Ways}

A large reduction in the magnitude of the generated membrane potential was observed in the IBG-treated group, compared to that of the untreated cells and cells in the presence of ampicillin (Figure 2A). Therefore, IBG involved disruption of the inner membrane of bacteria. The membrane disruption properties of IBG were monitored by the SYTOX Green uptake. The fluorescence value clearly increased treated with IBG, even at a concentration of $1 \times$ MIC, which indicated IBG could cause 
TABLE 1 | Activity of IBG against Staphylococcus aureus.

\begin{tabular}{lcc}
\hline Organism & IBG MIC $(\mu \mathbf{g} / \mathbf{m l})$ & VAN MIC $(\mu \mathbf{g} / \mathbf{m l})$ \\
\hline S. aureus ATCC 29213 (MSSA) & 4 & 1 \\
S. Aureus 29213 + 10\% FBS & 32 & 1 \\
MRSA ATCC 43300 & 4 & 1 \\
MRSA 43300 + 10\% FBS & 32 & 1 \\
Clinical MSSA (50) & $0.125-4$ & $0.5-1$ \\
Clinical MRSA (55) & $2-4$ & $0.5-1$ \\
\hline
\end{tabular}

IBG, isopropoxy benzene guanidine; VAN, vancomycin; FBS, fetal bovine serum; -, undetected. MIC, Minimum inhibitory concentration; MSSA, methicillin-sensitive S. aureus; MRSA, methicillin-resistant S. aureus.

TABLE 2 | MBC of IBG against Staphylococcus aureus.

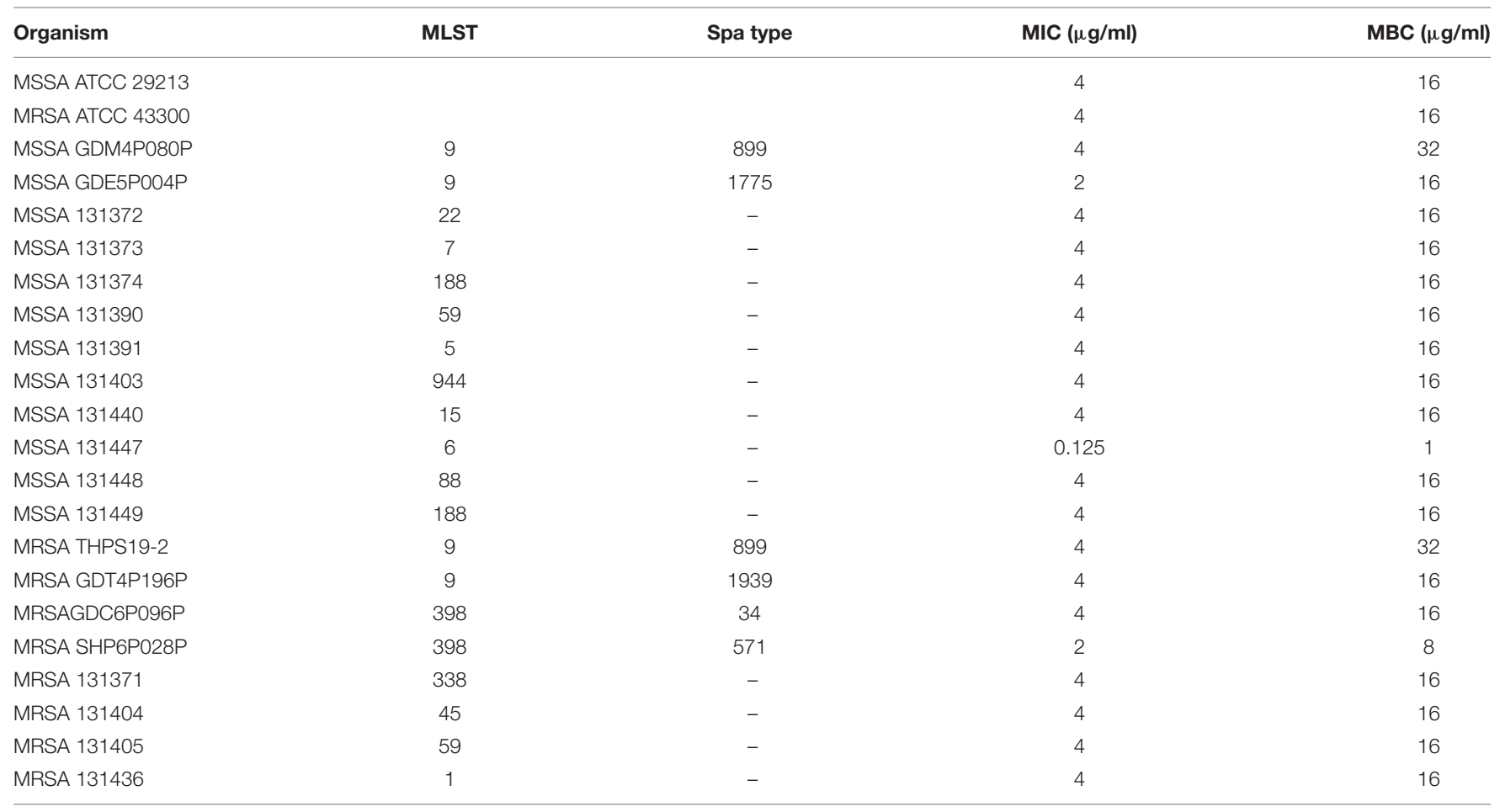

MBC, Minimum bactericidal concentration; IBG, isopropoxy benzene guanidine; -, undetected; MIC, Minimum inhibitory concentration; MSSA, methicillin-sensitive S. aureus; MRSA, methicillin-resistant S. aureus.

the damage of cell plasma membrane (Figure 2B). TEM results showed that IBG treatment caused remarkable morphological changes in S. aureus cells, such as mesosome-like structures, cells with ruptured wall and cytoplasmic membrane were found. Even worse, the cytoplasmic contents of some cells were released to the extracellular medium (Figure 2C).

\section{IBG Regulated the Expression of Membrane Related Genes}

The principal component analysis (PCA) analysis revealed that IBG successfully separated treated samples from untreated samples (Figure 3A). Compared to the control group, IBG treatment led to an up-regulation of 230 and down-regulation of 214 DEGs (Figure 3B). According to the GO and KEGG enrichment analysis, IBG treatment caused the changed expression of genes involved in basic metabolic processes, including purine metabolism, pyrimidine metabolism, amino sugar and nucleotide sugar metabolism, alanine, aspartate and glutamate metabolism (Figures 3C,D). Notably, more than a third of DEGs (159/444) located on cell membrane, which mostly related to membrane transport function (Figure 3E). These genes were associated with osmotolerance, including those encoding efflux pumps, enzymes involved in $\mathrm{ABC}$ transporters, cation transporters and phosphotransferase system (PTS) (Figures 4AC). Specifically, virulence, purine and pyrimidine biosynthesis pathway related genes in $S$. aureus were down-regulated under IBG treatment (Figures 4D-F).

\section{IBG Rescued Mice From MRSA Septicemic Infection}

Mice were infected with MRSA at a dose $\left(3.0 \times 10^{6} \mathrm{CFU} / \mathrm{mouse}\right)$ that led to a $83.3 \%$ mortality (Supplementary Figure 2). The 
A<smiles>CC(C)Oc1ccc(/C=N/NC(=N)N/N=C/c2ccc(OC(C)C)cc2)cc1</smiles>

B

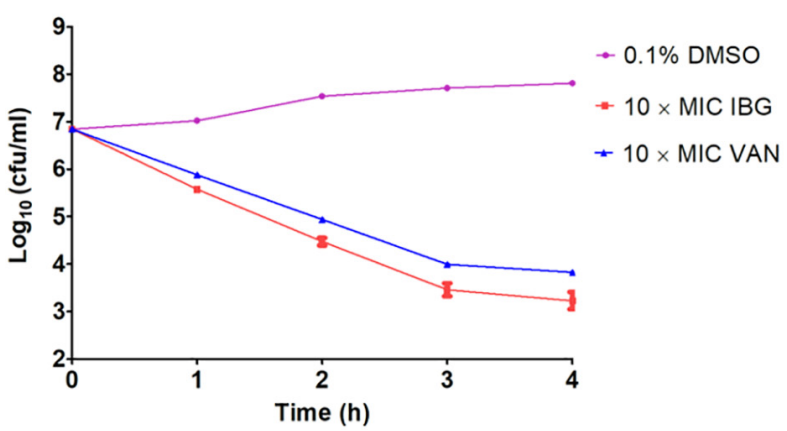

D

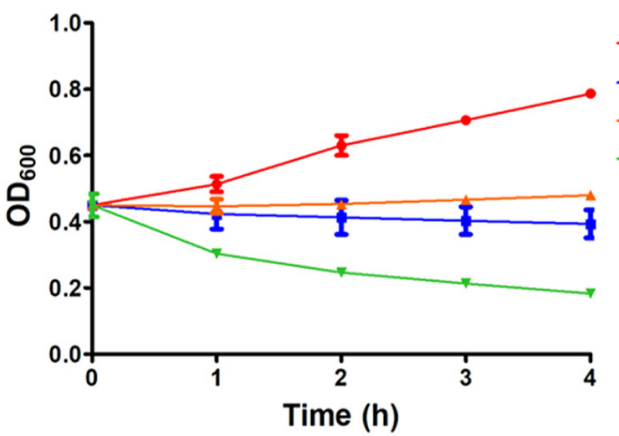

C

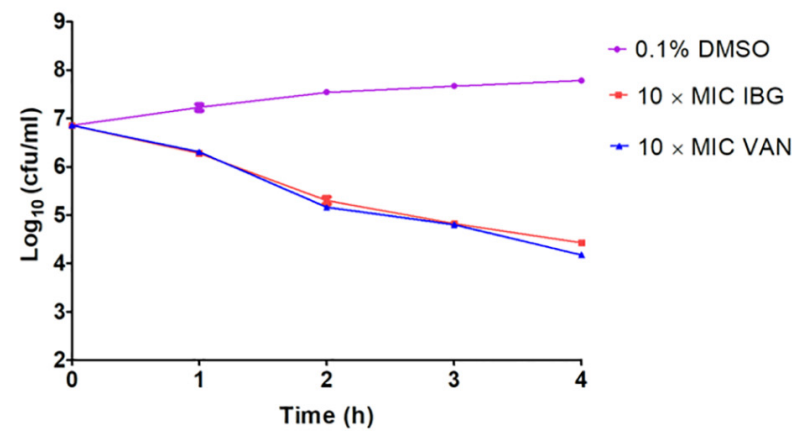

E

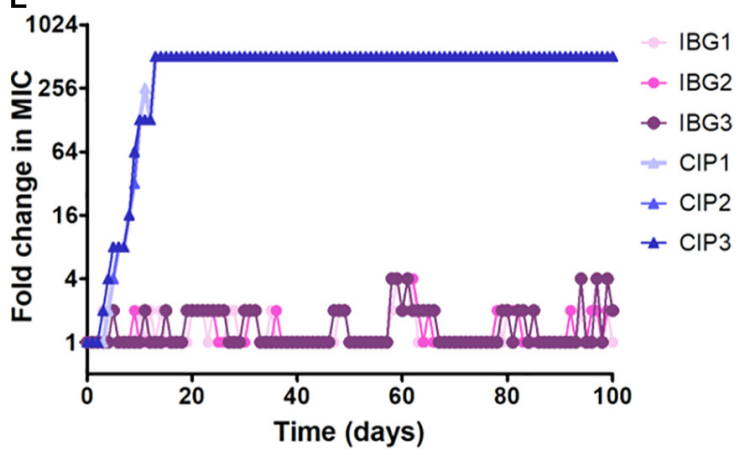

FIGURE 1 | IBG inhibits S. aureus growth without detectable mutant development. (A) Structure of IBG. (B) Viability of S. aureus ATCC 29213 treated with IBG or VAN. (C) Viability of MRSA ATCC 43300 treated with IBG or VAN for 4 h. (D) IBG treatment could not result in S. aureus ATCC 29213 lysis; (E) Appearance of spontaneous IBG and CIP-resistant S. aureus mutants over 100 days of serial passages. IBG, isopropoxy benzene guanidine; VAN, vancomycin; CIP, ciprofloxacin; Individual data points ( $n=3$ biologically independent experiments) and mean \pm SD. are shown.

survival rate of infected mice was 66.6 and $83.3 \%$ treated with IBG or VAN, respectively. These results suggested that IBG was efficacious in protecting mice from septicemic MRSA infection.

\section{DISCUSSION}

Staphylococcus aureus infections pose a significant challenge to public health due to the diminishing arsenal of effective antibiotics available. The development of novel antibacterial discovery has not kept pace with the rapid emergence of bacterial resistance to numerous antibiotics. Some researchers have proved that repurposing drug was the less time-consuming and more financially way to identify new antibiotics (Austin and Gadhia, 2017). In this study, we found that a guanidine compound displayed potent bactericidal activity against $S$. aureus by targeting cell membrane.
We demonstrated that IBG possessed antibacterial activity against multi-resistant $S$. aureus, including MRSA. Compared to the robenidine analog $16\left(3-\mathrm{OCH}_{3}\right)$ and $26\left[4-\mathrm{CH}\left(\mathrm{CH}_{3}\right)_{2}\right]$ reported previously (Abraham et al., 2016), IBG only had the isopropoxy group [3-OCH$\left.\left(\mathrm{CH}_{3}\right)_{2}\right]$ replacement in benzene ring, but showed the most active against MRSA. This finding was consistent with the antimicrobial activity of IBG against Enterococci (Zhang et al., 2019). Additionally, IBG maintained their activity against $S$. aureus isolates exhibiting resistance to different class of antibiotics, which indicated cross-resistance between IBG and these antibiotics is unlikely to occur.

Antibiotic resistance can evolve through sequential accumulation of multiple mutations. Hence, we performed laboratory evolution experiments to assess the propensity of bacteria to develop drug resistance (Toprak et al., 2011). We found that MIC of IBG was almost unchanged over 100 days, whereas the MIC of CIP quickly increased, which is consistent with the report that quinolones can easily induce bacteria 
TABLE 3 | Antimicrobial susceptibility of S. aureus ATCC 29213 mutants isolated by serial passage for 100 days.

\begin{tabular}{|c|c|c|c|c|c|c|}
\hline \multirow[t]{2}{*}{ Mutant strain } & \multirow[t]{2}{*}{ Mutated gene } & \multicolumn{5}{|c|}{$\operatorname{MIC}(\mu \mathrm{g} / \mathrm{ml})$} \\
\hline & & IBG & VAN & LNZ & CIP & GEN \\
\hline SP1-1 & & 4 & 1 & 1 & 0.25 & 0.5 \\
\hline SP1-58 & $\begin{array}{l}\text { pnp_1, atpE, walR, pcrA, ybiV, Org1_01939, Org1_01695, } \\
\text { Org1_01489 }\end{array}$ & 8 & 2 & 0.5 & 0.25 & 0.5 \\
\hline SP1-99 & $\begin{array}{l}\text { rsmG, atpE, dtd3,walR, pcrA, Org1_01695, Org1_01489, prkC, } \\
\text { lip2, mnhG1 }\end{array}$ & 8 & 2 & 0.5 & 0.5 & 0.5 \\
\hline SP2-1 & & 4 & 1 & 1 & 0.25 & 0.5 \\
\hline SP2-58 & $\begin{array}{l}\text { rpoE, atpE, walR, pcrA, ybiV, Org1_01695, Org1_01701, } \\
\text { Org1_01489, Org1_01939, Org1_00143, pnp_1 }\end{array}$ & 16 & 2 & 0.5 & 0.5 & 0.5 \\
\hline SP2-100 & nusG, atpE, pcrA, isdG_2, Org1_00312 & 8 & 2 & 1 & 0.25 & 0.5 \\
\hline SP3-1 & & 4 & 1 & 1 & 0.25 & 0.5 \\
\hline SP3-34 & atpE, walR, pcrA, ybiV, Org1_01489, lip2 & 8 & 2 & 1 & 0.5 & 0.5 \\
\hline SP3-66 & $\begin{array}{l}\text { Org1_00874, atpE, Org1_00657, walR, pcrA, ybiV, Org1_01695, } \\
\text { Org1_01489, Org1_01939, Org1_00143, pnp_1 }\end{array}$ & 8 & 2 & 0.5 & 0.25 & 0.5 \\
\hline SP3-100 & $\begin{array}{l}\text { Org1_00874, atpE, Org1_00657, walR, pcrA, ybiV, sasA, } \\
\text { Org1_01939, Org1_00143, tcaR, pnp_1, glpK }\end{array}$ & 8 & 2 & 0.5 & 0.25 & 0.5 \\
\hline
\end{tabular}

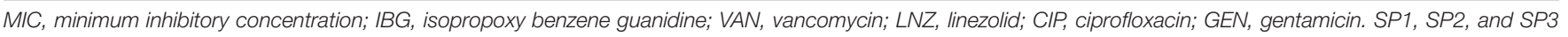
indicate three independent S. aureus 29213 cultures. Kb, S. aureus 29213 cells treated with 0.1\% DMSO.
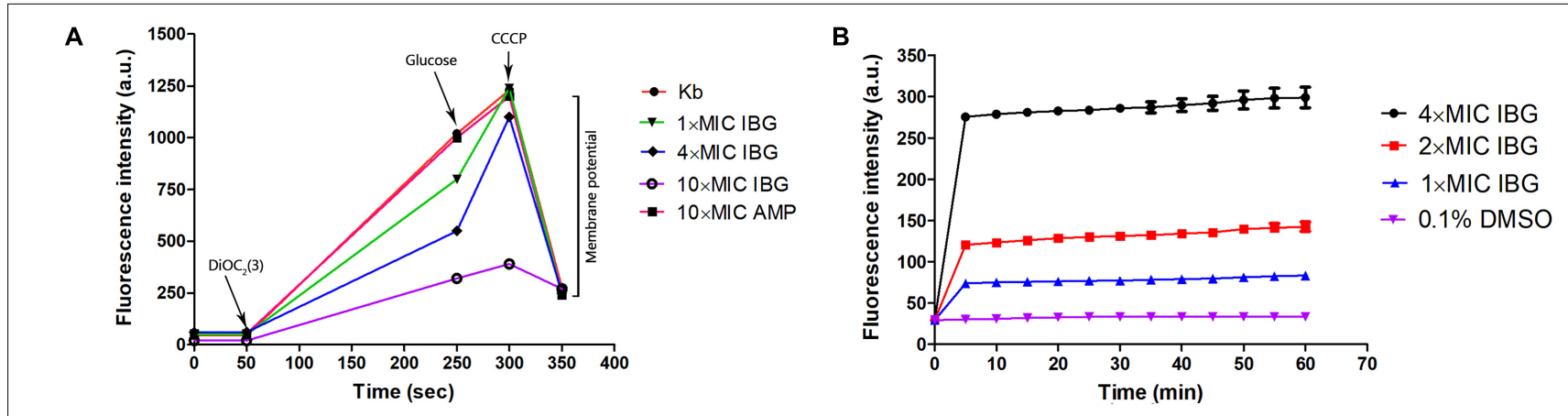

C
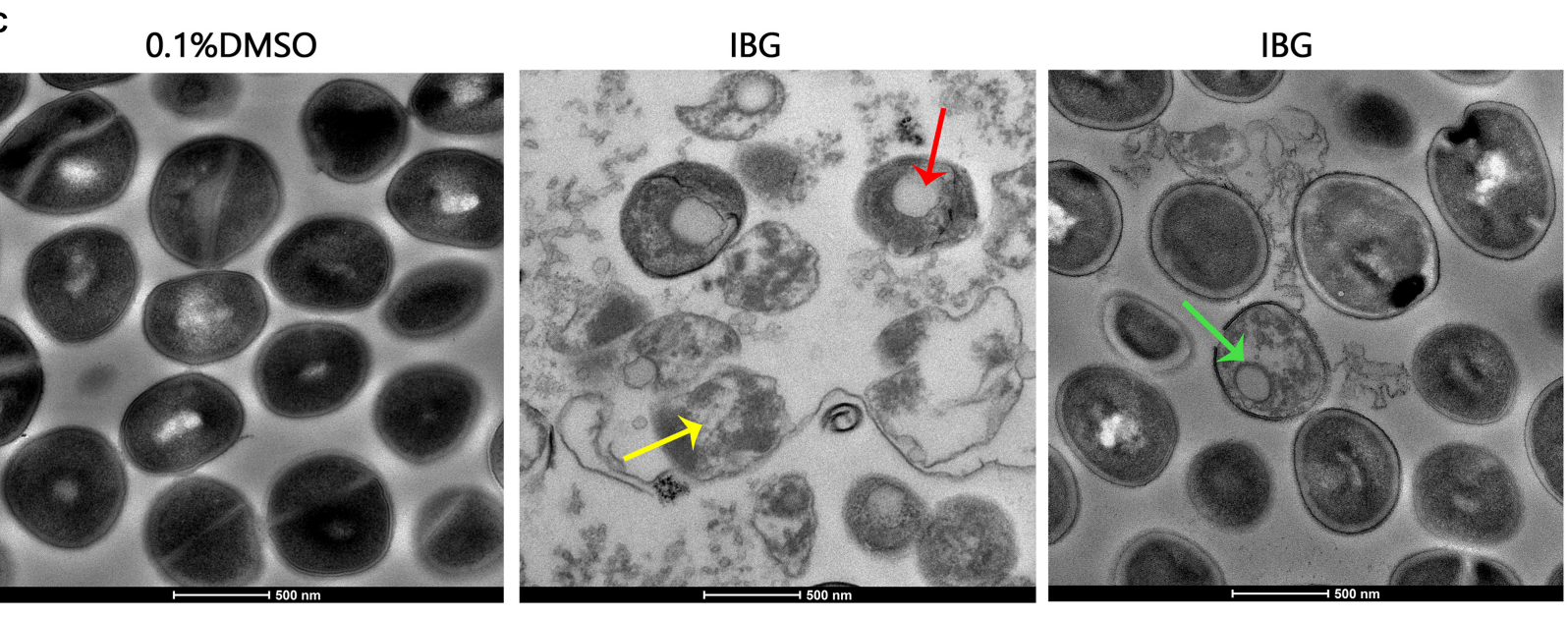

FIGURE 2 | IBG exerts its antibacterial action on the cell membrane of S. aureus. (A) IBG dissipates the membrane potential of S. aureus ATCC 29213. $P$ values were determined using a non-parametric one-way ANOVA. $P$ values of $\left({ }^{\star} \leq 0.05\right)\left({ }^{\star \star} P \leq 0.01\right)$ are considered as significant. (B) Uptake of SYTOX Green by exponential-phase S. aureus ATCC 29213 cells treated with IBG. (C) Transmission electron micrographs of S. aureus cells in response to IBG. Scale bars, 500 nm. These arrows indicate damage to the cell membrane by IBG. Kb, S. aureus ATCC 29213 control cell treated with $0.1 \%$ DMSO. 
A

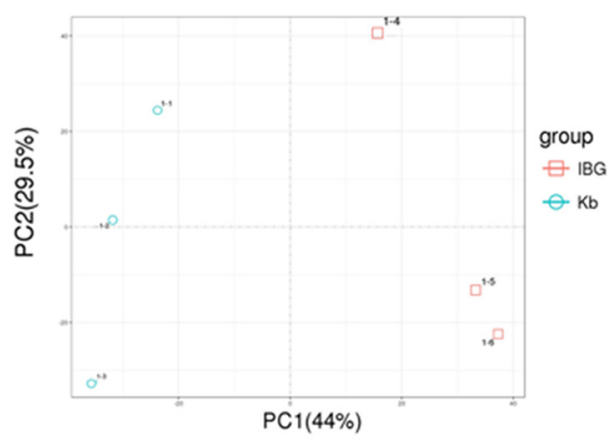

B

B Heatmap plot(Kb vs IBG)

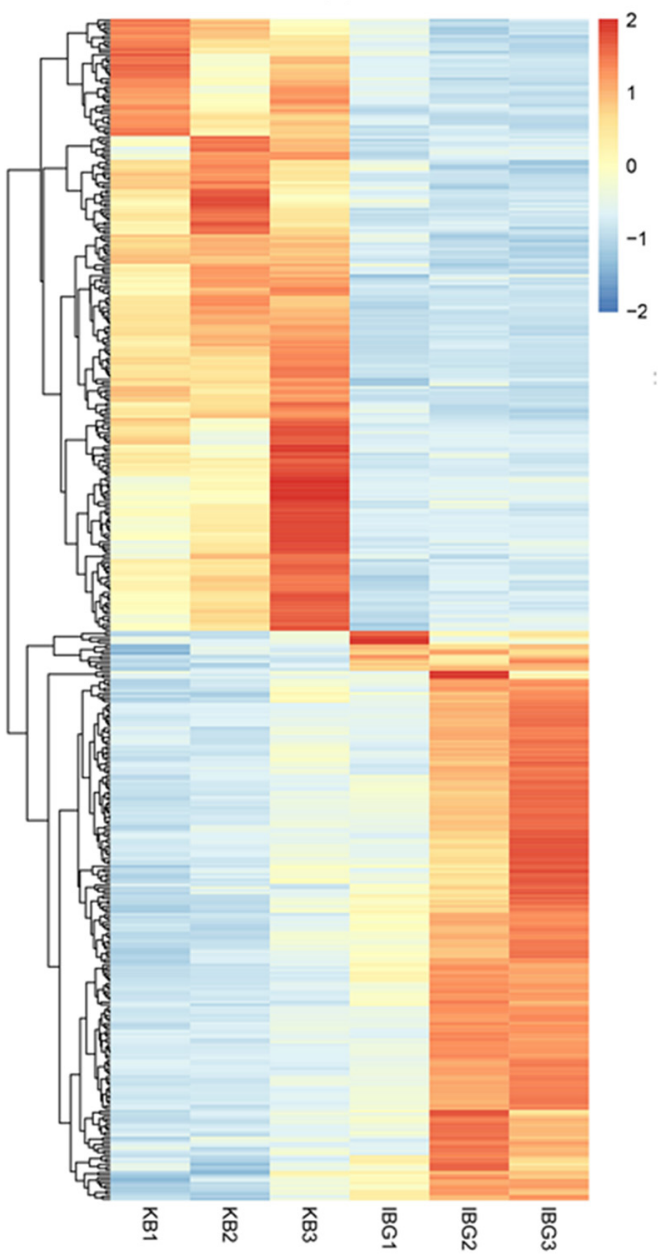

C

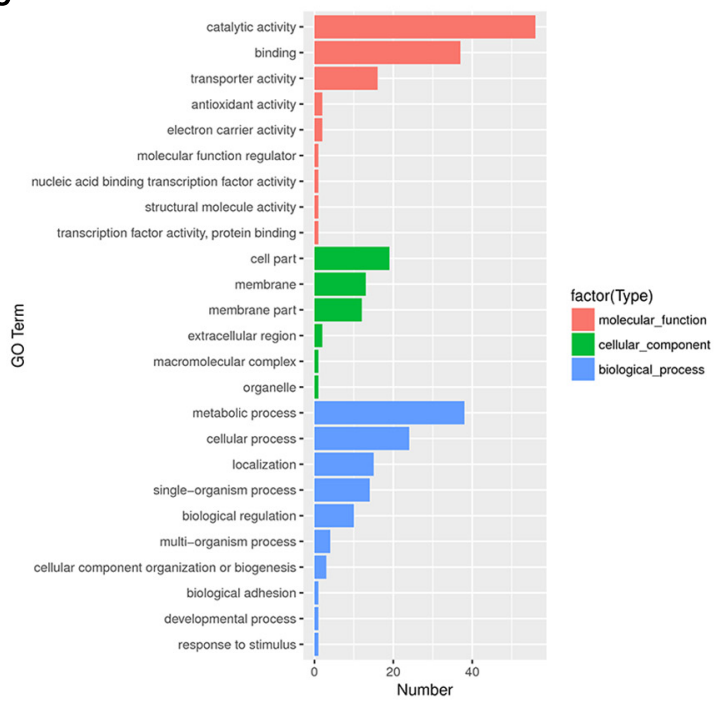

D

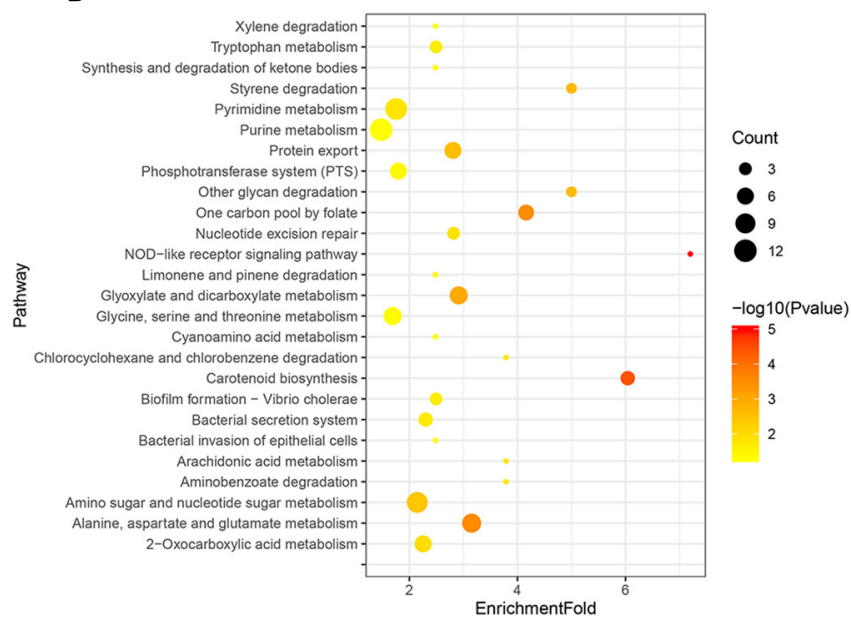

E

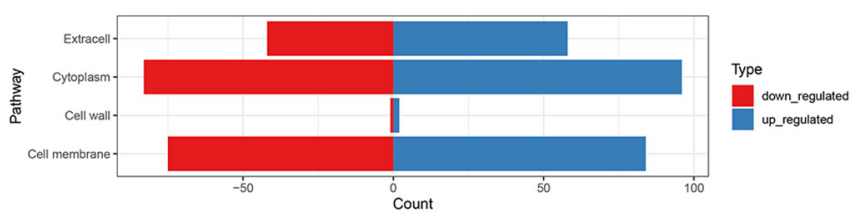

FIGURE 3 | Transcriptome analysis of DEGs in response to IBG stress. (A) PCA results. (B) Heat map representation of DEGs; (C) Go enrichment analysis of DEGs. (D) KEGG enrichment analysis of DEGs. (E) Subcellular localization of DEGs. DEGs, different expression genes; While all selected genes in RNA-seq data had FDR value of $<0.05$, which meant significant difference.

to develop drug resistance (Ling et al., 2015). These results demonstrated that $S$. aureus cannot easily develop resistance to IBG. To identify the genetic changes responsible for IBG resistance, we performed whole-genome sequence of seven hyposensitive $S$. aureus strains to IBG. All strains had two mutation genes (aptE and $p c r A$ ) and six of them had a mutation in walR gene. Gene atpE encodes subunit $\mathrm{C}$ of the ATP synthase which utilizes energy stored in the transmembrane electrochemical gradient to synthesize ATP and has been reported to involve in antibiotic resistance (Lamontagne Boulet et al., 2018). PcrA is an ATP-driven $3^{\prime}-5^{\prime}$ DNA helicase which is involved in DNA repair and plasmid rolling circle replication (Dillingham et al., 2001; Mhashal et al., 2016). Mutations in walR locus in S. aureus have been shown to relate to reduced 
A

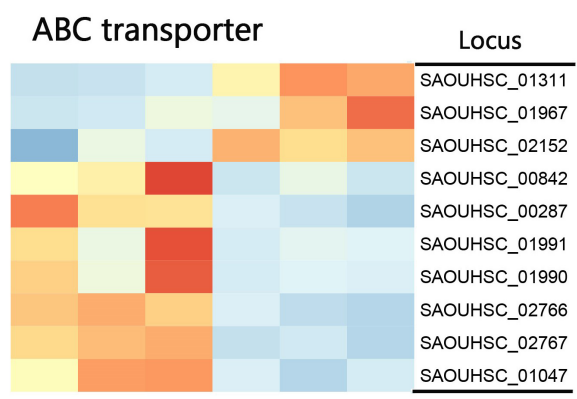

B Cation transporter

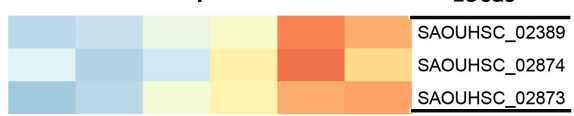

C Phosphotransferase system

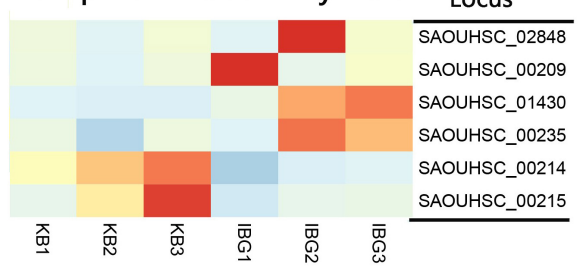

D Purine biosynthesis

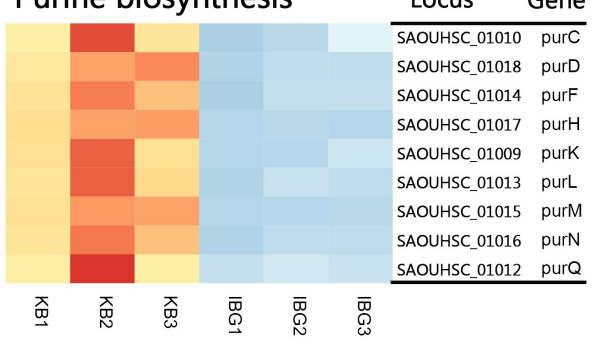

E Pyrimidine biosynthesis

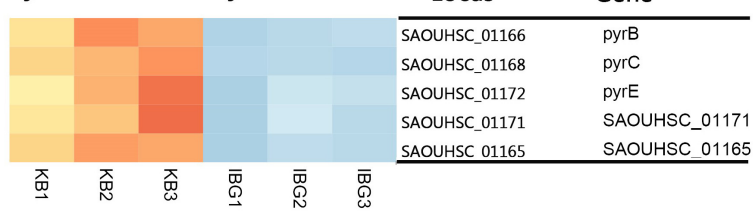

F Virulence

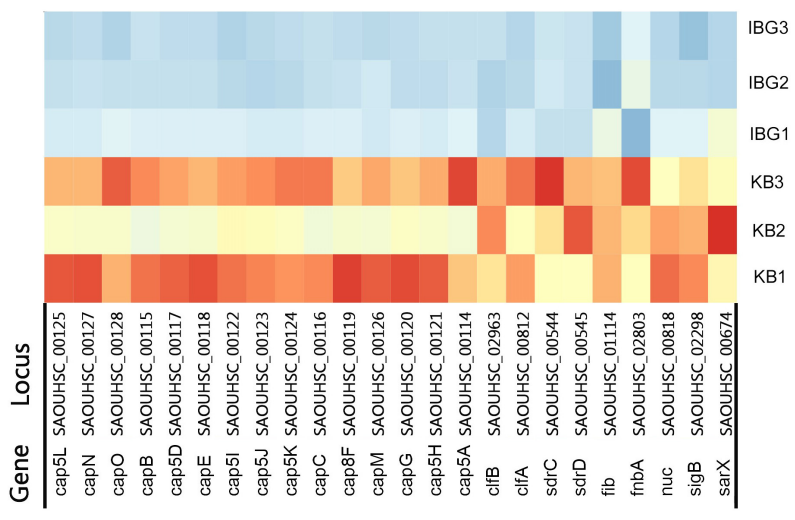

FIGURE 4 | Heat map representation of DEGs involved in main pathways. (A) DEGs related to ABC transporters. (B) DEGs involved in cation transporters; (C) DEGs related to phosphotransferase system. (D) DEGs involved in purine biosynthesis pathway. (E) DEGs involved in pyrimidine biosynthesis pathway. (F) DEGs involved in virulence. All genes represented have a > twofold change in expression treated with IBG relative to their expression in control samples.

susceptibility of vancomycin, which was also found in our study. WalR is a member of the two-component regulatory system WalKR that regulates genes involved in antibiotic resistance, autolysis, biofilm formation and cell wall metabolism (Howden et al., 2011). In addition, there is a $r p o E$ mutation in the day 34 of SP2. RpoE ( $\delta$ factor) is the DNA-dependent RNA polymerase (RNAP) subunit and works as a part of transcription machinery in $S$. aureus. It has been reported that $r p o E$ can be involved in orchestrating the ability of $S$. aureus to react and adapt to environmental changes and play a critical role in virulence (Weiss et al., 2014).

We previously confirmed that IBG displayed potent bactericidal activity against Enterococcus by disrupting the cell membrane potential ${ }^{12}$. This was also observed in $S$. aureus. The results showed IBG could disrupt the bacterial cell membranes. Similar findings were reported in a previous study in which MRSA strains were treated with robenidine analog NCL195 or techniques (Muthaiyan et al., 2012; Ogunniyi et al., 2017). Moreover, the micrograph indicated that the ultra-structural changes caused by IBG treatment might be irreversible. Perturbation of its structure by IBG maybe the crucial reason for its lethal action on S. aureus. However, death is not accompanied by a decline in culture absorbance.

Analysis of transcriptomic changes caused by IBG showed that the expression of genes associated with ABC transport and PTS transporter were remarkable changed. It was found that these transporter systems were associated with nutrient uptake and the export of toxins and antibiotics (Fleury et al., 2009; Liu M. et al., 2020). This may hint the disruption of the membrane potential could hinder the establishment and maintenance of essential energy sources for cell functioning. In addition, IBG treatment also repressed virulence and nucleotide biosynthesis. The downregulation of virulence factor genes including $\operatorname{sig} B$, capsules polysaccharides, might be very likely as a secondary effect for bacteria to survive (Shinji et al., 2011; Tuchscherr et al., 2015; Wang et al., 2019). Purine and pyrimidine biosynthesis pathway 
genes, crucial for cell growth via DNA and RNA synthesis, were down-regulated in IBG treatment group (Li et al., 2018). This was probably induced by the derived effects of increased membrane permeability. Its membrane-targeting actions toward bacteria might contribute to the lack of drug resistance during prolonged laboratory culture in its presence.

The effect with IBG was decreased at $10 \%$ FBS, which suggests a high level of protein binding of IBG. This was also observed in robenidine analogs (Abraham et al., 2016). But it does not necessarily render these compounds ineffective in vivo. Our results showed IBG could used as a therapeutic agent against mice MRSA systemic infection. Furthermore, IBG demonstrated additive activity when combined with antibiotics traditionally used to treat systemic MRSA infections. This is important given the emergence of resistance to systemic antimicrobials currently used in the clinic; pairing these antibiotics with IBG may improve the morbidity associated with bacterial infections and stymie the rate at which resistance to these antibiotics arises.

\section{CONCLUSION}

In the summary, we first identified that IBG possesses potent antimicrobial activity against clinical isolates of $S$. aureus by targeting cell membrane. IBG had a low probability of resistance selection and considerable efficacy. IBG has the promising potential to become a new class of antimicrobials for the treatment of Gram-positive bacterial infections.

\section{DATA AVAILABILITY STATEMENT}

The datasets presented in this study can be found in online repositories. The names of the repository/repositories and

\section{REFERENCES}

AbdelKhalek, A., Ashby, C. R. Jr., Patel, B. A., Talele, T. T., and Seleem, M. N. (2016). In vitro antibacterial activity of rhodanine derivatives against pathogenic clinical isolates. PLoS One 11:e164227. doi: 10.1371/journal.pone. 0164227

Abraham, R. J., Stevens, A. J., Young, K. A., Russell, C., Qvist, A., Khazandi, M., et al. (2016). Robenidine analogues as Gram-positive antibacterial agents. J. Med. Chem. 59, 2126-2138. doi: 10.1021/acs.jmedchem.5b01797

Antibiotics Currently in Global Clinical Development (2019). Tracking the Pipeline of Antibiotics in Development. Available online at: pewtrusts.org/antibioticpipeline

Årdal, C., Balasegaram, M., Laxminarayan, R., McAdams, D., Outterson, K., Rex, J. H., et al. (2019). Antibiotic development - economic, regulatory and societal challenges. Nat. Rev. Microbiol. 18, 267-274. doi: 10.1038/s41579-019-0293-3

Austin, B. A., and Gadhia, A. D. (2017). New therapeutic uses for existing drugs. Adv. Exp. Med. Biol. 1031, 233-247. doi: 10.1007/978-3-319-67144-4_14

Chou, K., and Shen, H. (2010). Cell-PLoc 2.0: an improved package of web-servers for predicting subcellular localization of proteins in various organisms. Nat. Sci. 02, 1090-1103. doi: 10.4236/ns.2010.210136

Clinical and Laboratory Standards Institute (2018). Performance Standards for Antimicrobial Susceptibility Testing, M100. 28th Edn. Wayne, PA: Clinical and Laboratory Standards Institute.

Dillingham, M. S., Soultanas, P., Wiley, P., Webb, M. R., and Wigley, D. B. (2001). Defining the roles of individual residues in the single-stranded DNA binding accession number(s) can be found in the article/Supplementary Material.

\section{ETHICS STATEMENT}

The animal study was reviewed and approved by the Animal Research Committee of South China Agricultural University (2018030). Animal studies were carried out at the animal laboratory center of South China Agricultural University.

\section{AUTHOR CONTRIBUTIONS}

ZZ conceived and designed the project. XZ and WX drafted the manuscript. XP and ZQ worked on the synthesis of compound IBG. YL and JH preformed the antimicrobial susceptibility tests, time-kill assay, and checkerboard assay. XZ carried out the RAN-Seq and data analysis. All authors read and approved the final manuscript.

\section{FUNDING}

The work was supported by the National Natural Science Foundation of China (Grant No. 31672608).

\section{SUPPLEMENTARY MATERIAL}

The Supplementary Material for this article can be found online at: https://www.frontiersin.org/articles/10.3389/fmicb. 2021.633467/full\#supplementary-material

site of PcrA helicase. Proc. Natl. Acad. Sci. U.S.A. 98, 8381-8387. doi: 10.1073/ pnas. 131009598

Falagas, M. E., Voulgaris, G. L., Tryfinopoulou, K., Giakkoupi, P., Kyriakidou, M., Vatopoulos, A., et al. (2019). Synergistic activity of colistin with azidothymidine against colistin-resistant Klebsiella pneumoniae clinical isolates collected from inpatients in Greek hospitals. Int. J. Antimicrob. Agents 53, 855-858. doi: 10. 1016/j.ijantimicag.2019.02.021

Fleury, B., Kelley, W. L., Lew, D., Gotz, F., Proctor, R. A., and Vaudaux, P. (2009). Transcriptomic and metabolic responses of Staphylococcus aureus exposed to supra-physiological temperatures. Int. J. Antimicrob. Agents 9:76. doi: 10.1186/ 1471-2180-9-76

Guan, D., Chen, F., Faridoon, Liu, J., Li, J., Lan, L., et al. (2018). Design and synthesis of pyrophosphate-targeting vancomycin derivatives for combating vancomycin-resistant Enterococci. Chemmedchem 13, 1644-1657. doi: 10.1002/ cmdc. 201800252

Howden, B. P., McEvoy, C. R., Allen, D. L., Chua, K., Gao, W., Harrison, P. F., et al. (2011). Evolution of multidrug resistance during Staphylococcus aureus infection involves mutation of the essential two component regulator WalKR. PLoS Pathog. 7:e1002359. doi: 10.1371/journal.ppat.1002359

Kim, W., Zhu, W., Hendricks, G. L., Van Tyne, D., Steele, A. D., Keohane, C. E., et al. (2018). A new class of synthetic retinoid antibiotics effective against bacterial persisters. Nature 556, 103-107. doi: 10.1038/nature26157

Lakhundi, S., and Zhang, K. (2018). Methicillin-resistant Staphylococcus aureus: molecular characterization, evolution, and epidemiology. Clin. Microbiol. Rev. 31, e18-e20. doi: 10.1128/CMR.00020-18 
Lamontagne Boulet, M., Isabelle, C., Guay, I., Brouillette, E., Langlois, J. P., Jacques, P. E., et al. (2018). Tomatidine is a lead antibiotic molecule that targets Staphylococcus aureus ATP synthase subunit c. Antimicrob. Agents Chemother. 62, e02197-17. doi: 10.1128/aac.02197-17

Li, J., Ahn, J., Liu, D., Chen, S., Ye, X., and Ding, T. (2016). Evaluation of ultrasound-induced damage to Escherichia coli and Staphylococcus aureus by flow cytometry and transmission electron microscopy. Appl. Environ. Microbiol. 82, 1828-1837. doi: 10.1128/aem.03080-15

Li, L., Abdelhady, W., Donegan, N. P., Seidl, K., Cheung, A., Zhou, Y. F., et al. (2018). Role of purine biosynthesis in persistent methicillin-resistant Staphylococcus aureus infection. J. Infect. Dis. 218, 1367-1377. doi: 10.1093/ infdis/jiy340

Ling, L. L., Schneider, T., Peoples, A. J., Spoering, A. L., Engels, I., Conlon, B. P., et al. (2015). A new antibiotic kills pathogens without detectable resistance. Nature 517, 455-459. doi: 10.1038/nature14098

Liu, M., Feng, M., Yang, K., Cao, Y., Zhang, J., Xu, J., et al. (2020). Transcriptomic and metabolomic analyses reveal antibacterial mechanism of astringent persimmon tannin against methicillin-resistant Staphylococcus aureus isolated from pork. Food Chem. 309:125692. doi: 10.1016/j.foodchem.2019.125692

Liu, Y., Jia, Y., Yang, K., Li, R., Xiao, X., Zhu, K., et al. (2020). Metformin restores tetracyclines susceptibility against multidrug resistant bacteria. $A d v$. Sci. 7:1902227. doi: 10.1002/advs.201902227

Massimba-Dibama, H., Mourer, M., Constant, P., Daffe, M., and Regnouf-deVains, J. B. (2015). Guanidinium compounds with sub-micromolar activities against Mycobacterium tuberculosis. Synth. Charact. Biol. Eval. 23, 5410-5418. doi: 10.1016/j.bmc.2015.07.053

Mhashal, A. R., Choudhury, C. K., and Roy, S. (2016). Probing the ATPinduced conformational flexibility of the PcrA helicase protein using molecular dynamics simulation. J. Mol. Model. 22:54. doi: 10.1007/s00894-016-2922-3

Mohammad, H., Reddy, P. V., Monteleone, D., Mayhoub, A. S., Cushman, M., and Seleem, M. N. (2015). Synthesis and antibacterial evaluation of a novel series of synthetic phenylthiazole compounds against methicillin-resistant Staphylococcus aureus (MRSA). Eur. J. Med. Chem. 94, 306-316. doi: 10.1016/j. ejmech.2015.03.015

Muthaiyan, A., Martin, E. M., Natesan, S., Crandall, P. G., Wilkinson, B. J., and Ricke, S. C. (2012). Antimicrobial effect and mode of action of terpeneless coldpressed Valencia orange essential oil on methicillin-resistant Staphylococcus aureus. J. Appl. Microbiol. 112, 1020-1033. doi: 10.1111/j.1365-2672.2012. 05270.x

Nambiar, S., Laessig, K., Toerner, J., Farley, J., and Cox, E. (2014). Antibacterial drug development: challenges, recent developments, and future considerations. Clin. Pharmacol. Ther. 96, 147-149. doi: 10.1038/clpt.2014.116

Ogunniyi, A. D., Khazandi, M., Stevens, A. J., Sims, S. K., Page, S. W., Garg, S., et al. (2017). Evaluation of robenidine analog NCL195 as a novel broad-spectrum antibacterial agent. Plos One 12:e0183457. doi: 10.1371/journal.pone.0183457

Okano, A., Isley, N. A., and Boger, D. L. (2017). Peripheral modifications of $[\Psi[\mathrm{CH}(2) \mathrm{NH}] \mathrm{Tpg}(4)]$ vancomycin with added synergistic mechanisms of action provide durable and potent antibiotics. Proc. Natl. Acad. Sci. U.S.A. 114, E5052-E5061. doi: 10.1073/pnas.1704125114

Pushpakom, S., Iorio, F., Eyers, P. A., Escott, K. J., Hopper, S., Wells, A., et al. (2019). Drug repurposing: progress, challenges and recommendations. Nat. Rev. Drug Discov. 18, 41-58. doi: 10.1038/nrd.2018.168

Saczewski, F., and Balewski, L. (2013). Biological activities of guanidine compounds, 2008 - 2012 update. Expert Opin. Ther. Pat. 23, 965-995. doi: $10.1517 / 13543776.2013 .788645$

Shinji, H., Yosizawa, Y., Tajima, A., Iwase, T., Sugimoto, S., Seki, K., et al. (2011). Role of fibronectin-binding proteins $\mathrm{A}$ and $\mathrm{B}$ in in vitro cellular infections and in vivo septic infections by Staphylococcus aureus. Infect. Immun. 79, 2215-2223. doi: 10.1128/iai.00133-11

Tacconelli, E., Carrara, E., Savoldi, A., Harbarth, S., Mendelson, M., Monnet, D. L., et al. (2018). Discovery, research, and development of new antibiotics: the WHO priority list of antibiotic-resistant bacteria and tuberculosis. Lancet Infect. Dis. 18, 318-327. doi: 10.1016/s1473-3099(17) 30753-3

Talbot, G. H., Jezek, A., Murray, B. E., Jones, R. N., Ebright, R. H., Nau, G. J., et al. (2019). The infectious diseases society of America's 10 x '20 initiative (10 new systemic antibacterial agents US food and drug administration approved by 2020): is 20 x '20 a possibility? Clin. Infect. Dis. 69, 1-11. doi: 10.1093/cid/ ciz089

Thangamani, S., Mohammad, H., Abushahba, M. F., Sobreira, T. J., Hedrick, V. E., Paul, L. N., et al. (2016). Antibacterial activity and mechanism of action of auranofin against multi-drug resistant bacterial pathogens. Sci. Rep. 6:22571. doi: 10.1038/srep22571

Toprak, E., Veres, A., Michel, J., Chait, R., Hartl, D. L., and Kishony, R. (2011). Evolutionary paths to antibiotic resistance under dynamically sustained drug selection. Nat. Genet. 44, 101-105. doi: 10.1038/ng.1034

Tuchscherr, L., Bischoff, M., Lattar, S. M., Noto Llana, M., Pfortner, H., Niemann, S., et al. (2015). Sigma factor SigB is crucial to mediate Staphylococcus aureus adaptation during chronic infections. PLoS Pathog. 11:e1004870. doi: 10.1371/ journal.ppat.1004870

Turner, N. A., Sharma-Kuinkel, B. K., Maskarinec, S. A., Eichenberger, E. M., Shah, P. P., Carugati, M., et al. (2019). Methicillin-resistant Staphylococcus aureus: an overview of basic and clinical research. Nat. Rev. Microbiol. 17, 203-218. doi: 10.1038/s41579-018-0147-4

Wang, X., Wu, H., Niu, T., Bi, J., Hou, H., Hao, H., et al. (2019). Downregulated expression of virulence factors induced by benzyl isothiocyanate in Staphylococcus aureus: a transcriptomic analysis. Int. J. Mol. Sci. 20:5441. doi: 10.3390/ijms20215441

Wang, Y., Mowla, R., Guo, L., Ogunniyi, A. D., Rahman, T., De Barros Lopes, M. A., et al. (2017). Evaluation of a series of 2-napthamide derivatives as inhibitors of the drug efflux pump AcrB for the reversal of antimicrobial resistance. Bioorg. Med. Chem. Lett. 27, 733-739. doi: 10.1016/j.bmcl.2017.01. 042

Weiss, A., Ibarra, J. A., Paoletti, J., Carroll, R. K., and Shaw, L. N. (2014). The delta subunit of RNA polymerase guides promoter selectivity and virulence in Staphylococcus aureus. Infect. Immun. 82, 1424-1435. doi: 10.1128/iai.01508-14

Zhang, X., Han, D., Pei, P., Hao, J., Lu, Y., Wan, P., et al. (2019). In vitro antibacterial activity of isopropoxy benzene guanidine against multidrugresistant Enterococci. Infect. Drug Resist. 12, 3943-3953. doi: 10.2147/IDR. S234509

Conflict of Interest: XP and ZQ was employed by company Guangzhou Insighter Biotechnology Co., Ltd.

The remaining authors declare that the research was conducted in the absence of any commercial or financial relationships that could be construed as a potential conflict of interest.

Copyright (C) 2021 Zhang, Xiong, Peng, Lu, Hao, Qin and Zeng. This is an open-access article distributed under the terms of the Creative Commons Attribution License (CC BY). The use, distribution or reproduction in other forums is permitted, provided the original author(s) and the copyright owner(s) are credited and that the original publication in this journal is cited, in accordance with accepted academic practice. No use, distribution or reproduction is permitted which does not comply with these terms. 